\title{
Cadaveric Dissection a Thing of the Past? The Insight of Consultants, Fellows, and Residents
}

Haider Ghazanfar ${ }^{1}$, Sannah Rashid ${ }^{2}$, Ashraf Hussain ${ }^{3}$, Madiha Ghazanfar ${ }^{4}$, Ali Ghazanfar ${ }^{5}$, Arshad Javaid ${ }^{3}$

1. Internal Medicine, Shifa College of Medicine, Islamabad, PAK 2. Pediatrics, Shifa College of Medicine, Islamabad, PAK 3. Department of Anatomy, Shifa College of Medicine, Islamabad, PAK 4. Emergency Medicine, Shifa International Hospital, Islamabad, PAK 5. Federal Medical and Dental College, Islamabad, PAK

$\square$ Corresponding author: Haider Ghazanfar, haidergh@gmail.com

Disclosures can be found in Additional Information at the end of the article

\section{Abstract}

\section{Objective}

The objective of our study was to determine whether cadaveric dissection is a necessity in medical education. Another purpose of our study was to assess the attitude and perception of consultants, residents, and fellows about cadaveric dissection and whether it helped them in their medical practices.

\section{Method}

We performed an analytical cross-sectional study among consultants, fellows, and residents of different specialty areas practicing in Punjab. A self-constructed questionnaire compromising of 41 items was used to assess the perception of doctors about cadaveric dissection and other alternative anatomy teaching methods. Consultants, fellows, and residents who were in clinical practice for more than six months were included in the study.

\section{Results}

Out of the total sample size of $842,44.7 \%$ were female medical doctors and $55.3 \%$ were male medical doctors. Cadaveric dissection was thought to be the most effective method for teaching anatomy by $27.9 \%$ of the doctors. Mean cadaveric dissection, prosection and didactic teaching components were scored significantly higher by doctors in surgery and allied fields $(\mathrm{p}<0.001)$. Doctors in the surgical and allied field were 0.55 times less likely to think that cadaveric dissection was unethical as compared to doctors working in medicine and allied fields $(\mathrm{p}<0.001)$.

Received 03/28/2018

Review began 03/28/2018

Review ended 03/28/2018

Published 04/03/2018

C) Copyright 2018

Ghazanfar et al. This is an open access article distributed under the terms of the Creative Commons Attribution License CC-BY 3.0., which permits unrestricted use, distribution, and reproduction in any medium, provided the original author and source are credited.

\section{Conclusion}

Dissection is still considered by several doctors as a valuable source of learning anatomy. However, the future of teaching anatomy does not depend on any single method. It is, in fact, the right combination of all available resources and using them in an interactive way that maximizes outcomes.

Categories: Family/General Practice, Medical Education, Quality Improvement 
Keywords: perception, anatomy, dissection, female, physician

\section{Introduction}

Anatomy is derived from the Greek word "anatome” which means cutting up. It is the branch of science that studies the structure of the body. Over the past couples of decades, significant advances have occurred in the field of anatomy. Anatomy is one of the core basic science subjects studied by medicals student all across the world when they begin their medical education.

Traditionally, block system of education has been used in medical colleges to teach anatomy along with other core basic science subjects. Cadaveric dissection has been used for centuries for teaching gross anatomy all over the world [1]. Cadaveric dissection has been considered a necessity in the learning of gross anatomy and thought to contribute significantly to a future professional career [2-4]. Cadaveric dissection helps medical students by helping them in understanding the three-dimensional relationship of different anatomical structures and appreciating anatomical variations [5]. It has become a greatly acknowledged fact that good medical or surgical practice could only be based on adequate anatomical knowledge of human anatomy which can only be learned from cadaveric dissection [6].

Medical education's paradigm has shifted towards problem-based learning and integrated curriculum [7] This has resulted in the studying of core basic science subjects including anatomy in the light of clinical context only and the abandoning of cadaveric dissection. In most institutions, gross anatomy knowledge is now taught via a small group discussion based on various clinical cases which are followed by a whole class lecture session [8-9].

The paucity of cadavers and high financial cost have significantly contributed to the development of alternative teaching techniques; consequently leading to more medical students having no experience of cadaveric dissection. Furthermore, advances in web-based medical technology have resulted in the development of virtual dissection programs. These programs have been found to be an effective way teaching anatomy [10] and are being preferred over cadaveric dissection, as its use is not associated with emotional and ethical issues [11].

Alternative teaching methods are now more commonly deployed in teaching gross anatomy. This issue will become even more common with the passage of time. The age-old debate of 'to dissect, or nor to dissect' still rages on. The majority of medical students don't do cadaveric dissection nowadays. Does this mean these medical students won't become better doctors? Is cadaveric dissection simply a rite of passage or is it a necessity? The aim of our study was to determine whether cadaveric dissection is a necessity in medical education and can it be replaced by newer alternative techniques. Another purpose of our study was to assess the attitude and perception of consultants, residents, and fellows about cadaveric dissection and whether it helped them in their medical practices.

\section{Materials And Methods}

We performed an analytical cross-sectional study among consultants, fellows and residents of different specialties practicing in Rawalpindi, Islamabad, Wah, Multan, Bahawalpur, and Lahore from January 2016 to April 2017. A self-constructed questionnaire compromising of 41 items was used to assess the perception of consultants, fellows, and residents about cadaveric dissection and other alternative anatomy teaching methods. Consultants, fellows, and residents who were in clinical practice for more than six months were included in the study. Participants were chosen by stratified randomized sampling. The sample size of 802 was calculated by using the World Health Organization (WHO) sample size calculator, keeping 2.25 as absolute precision required, the prevalence of cadaveric dissection at $88 \%$, and the 
confidence level at 95. A total of 1000 forms were given out of which $84.2 \%$ (842) were completely filled. Each participant was given 10 minutes to fill the form which was followed by a 10-minute interview. Informed consent was taken from all the participants and the identity of the respondents was kept anonymous.

SPSS version 21 was used to analyze the data. After the responses of the consultants, fellows and residents were gathered, the internal validity of the questionnaire was tested using Cronbach's alpha. The total questionnaire Cronbach's alpha value was calculated as 0.712 providing evidence for the acceptable internal validity of the questionnaire.

Mean and the standard deviation was calculated for quantitative variables like age and the question addressing how well the teaching aim was achieved. Frequency and percentage were calculated for qualitative variables like gender, specialty type, and questions related to the perception of cadaveric dissection. Independent $t$-test was performed to find the association between specialty type and the question addressing the perception of the consultant. A p-value of less than 0.05 was considered as significant.

\section{Results}

Mean age of the participants was $34.78 \pm 8.70$. Out of the total sample size of $842 ; 44.7 \%$ (376) were female medical doctors and 55.3\% (466) were male medical doctors. About $41.8 \%$ of the doctors were in surgery and allied and $58.2 \%$ of the doctors were in medicine and allied. About 411 (48.8\%) of the doctors were working in private hospital while 431 (51.2\%) of the doctors were working in a government hospital. About 349 (41.4\%) of the participants were consultants while 493 (58.6\%) of the participants were fellow and residents.

Cadaveric dissection was thought to be the most effective method for teaching anatomy by 27.9\% (235) of the doctors. About 11.5\% (97) doctors had not performed cadaveric dissection during the undergraduate program while $88.5 \%$ (745) had performed cadaveric dissection at least once during the undergraduate program. About $58.1 \%$ of the doctors were of the opinion that cadaveric dissection can be replaced by alternative teaching method. About $47.4 \%$ doctors were of the opinion that cadaveric dissection in an undergraduate program can help in their current specialty. Around 43.3\% doctors disagreed with the statement that cadaveric dissection is must for every doctor. Only $3.3 \%$ doctors thought that cadaveric dissection is a waste of time. Around 47.5\% doctors thought that cadaveric dissection is unethical. About $56.3 \%$ doctors believed that cadaveric dissection was religiously acceptable. Around $44.7 \%$ doctors thought that cadaveric dissection was worth the cost spent on it.

About 64.0\% doctors agreed that it was possible to practice their current specialty without doing cadaveric dissection in the undergraduate program. About $28.7 \%$ doctors thought that cadaveric dissection helps in clearing post-graduate examinations. Around $28.7 \%$ doctor thought that cadaveric dissection helps a person in becoming a better doctor. Only $22.4 \%$ of the doctors thought that cadaveric dissection helps in diagnosing diseases. While Only $13.4 \%$ doctors were of the opinion that cadaveric dissection helps in deciding which specialty to opt for and only $8.8 \%$ doctors reported that cadaveric dissection helped them in attaining the current position.

The live surgeries method had the highest score in the method of teaching anatomy while didactic teaching method had the lowest score. Mean total score of each method of teaching anatomy has been shown in Table 1 . 


\section{Cureus}

\section{Method of Teaching Anatomy}

Cadaveric Dissection

Cadaveric Prosection

Model

Computer Model Programs

Live Surgeries

Didactic Teaching
Total Mean \pm SD

$2.90 \pm 1.01$

$2.78 \pm 0.91$

$3.00 \pm 0.89$

$2.86 \pm 1.00$

$3.44 \pm 0.73$

$2.42 \pm 0.86$

\section{TABLE 1: Mean Total Score of Teaching Methods}

Independent sample t-test was applied to assess the association between type of specialty field and scores of different categories of teaching anatomy. Mean cadaveric dissection, prosection, and didactic teaching components were scored significantly higher by doctors in surgery and allied field while live surgeries component was scored significantly higher by doctors in medicine and allied field $(\mathrm{p}<0.001)$. This has been shown in Table 2 .

\begin{tabular}{|c|c|c|c|c|}
\hline & Surgery and Allied & Medicine and Allied & & \\
\hline Method of Teaching Anatomy & Total Mean \pm SD & Total Mean \pm SD & $\mathbf{t}$ & p-value \\
\hline Cadaveric Dissection & $3.17 \pm 1.12$ & $2.70 \pm 0.88$ & 6.834 & $<0.001$ \\
\hline Cadaveric Prosection & $3.11 \pm 0.85$ & $2.54 \pm 0.88$ & 9.294 & $<0.001$ \\
\hline Model & $3.05 \pm 0.90$ & $3.08 \pm 0.88$ & -0.564 & 0.573 \\
\hline Computer Model Programs & $2.83 \pm 1.08$ & $2.88 \pm 0.96$ & -0.646 & 0.518 \\
\hline Live Surgeries & $3.26 \pm 0.64$ & $3.57 \pm 0.77$ & -6.207 & $<0.001$ \\
\hline Didactic Teaching & $2.62 \pm 0.70$ & $2.28 \pm 0.94$ & 5.615 & $<0.001$ \\
\hline
\end{tabular}

TABLE 2: Association Between Type of Specialty and Scores of Different Categories of Teaching Anatomy

Independent sample t-test was applied to assess the association between Type of hospital and scores of different categories of teaching anatomy. Mean cadaveric dissection, prosection, model, computer model programs, and didactic teaching components were scored significantly higher by doctors working in private hospitals while live surgeries component was scored significantly higher by doctors in public hospitals $(\mathrm{p}<0.001)$. This has been shown in Table 3 . 


\section{Cureus}

\begin{tabular}{|c|c|c|c|c|}
\hline & Private Hospital & Public Hospital & & \\
\hline Method of Teaching Anatomy & Total Mean \pm SD & Total Mean \pm SD & $\mathbf{t}$ & p-value \\
\hline Cadaveric Dissection & $3.62 \pm 0.77$ & $2.21 \pm 0.69$ & 27.959 & $<0.001$ \\
\hline Cadaveric Prosection & $3.42 \pm 0.64$ & $2.17 \pm 0.69$ & 27.198 & $<0.001$ \\
\hline Model & $3.47 \pm 0.611$ & $2.69 \pm 0.95$ & 14.297 & $<0.001$ \\
\hline Computer Model Programs & $3.54 \pm 0.66$ & $2.20 \pm 0.84$ & 25.637 & $<0.001$ \\
\hline Live Surgeries & $3.34 \pm 0.80$ & $3.53 \pm 0.65$ & -3.678 & $<0.001$ \\
\hline Didactic Teaching & $2.75 \pm 0.90$ & $2.11 \pm 0.70$ & 11.601 & $<0.001$ \\
\hline
\end{tabular}

TABLE 3: Association Between Type of Hospital and Scores of Different Categories of Teaching Anatomy

Independent sample t-test was applied to assess the association between gender and scores of different categories of teaching anatomy. Mean cadaveric dissection, prosection and models were scored significantly higher by the female while computer model programs, Live surgeries, and Didactic teaching components were scored significantly higher by male doctors $(\mathrm{p}<0.05)$. This has been shown in Table 4.

\begin{tabular}{|c|c|c|c|c|}
\hline & Male & Female & & \\
\hline Method of I eaching Anatomy & lotal Mean \pm SD & I otal Mean $\pm S D$ & $\mathbf{t}$ & p-value \\
\hline Cadaveric Dissection & $2.74 \pm 0.97$ & $3.09 \pm 1.03$ & -4.999 & $<0.001$ \\
\hline Cadaveric Prosection & $2.58 \pm 0.89$ & $3.03 \pm 0.88$ & -7.235 & $<0.001$ \\
\hline Model & $2.91 \pm 1.00$ & $3.27 \pm 0.70$ & -5.807 & $<0.001$ \\
\hline Computer Model Programs & $2.96 \pm 1.08$ & $2.74 \pm 0.90$ & 3.195 & 0.001 \\
\hline Live Surgeries & $3.55 \pm 0.62$ & $3.30 \pm 0.83$ & 5.226 & $<0.001$ \\
\hline Didactic Teaching & $2.56 \pm 1.03$ & $2.25 \pm 0.55$ & 5.262 & $<0.001$ \\
\hline
\end{tabular}

TABLE 4: Association Between Gender and Scores of Different Categories of Teaching Anatomy

Pearson Correlation was done to assess for an association between age and scores of a different method of teaching anatomy. Pearson correlation showed significant positive correlation between age and cadaveric dissection method $\mathrm{p}<0.0005(\mathrm{r}=0.152)$, cadaveric prosection $\mathrm{p}<0.0005(\mathrm{r}=0.125)$, age Models $\mathrm{p}<0.0005(\mathrm{r}=0.318)$, computer model program $\mathrm{p}<0.005(\mathrm{r}=0621)$ 
and didactic teaching $\mathrm{p}<0.005(\mathrm{r}=0.405)$. Pearson correlation showed a negative correlation between age and live surgeries $\mathrm{p}=0.008(\mathrm{r}=-0.091)$.

Pearson chi-square test was applied to assess the association between gender, type of specialty and type of Hospital with "do you think Cadaveric Dissection is unethical". Doctors in surgical and allied field were 0.55 times less likely to think that cadaveric dissection was unethical as compared to doctors working in medicine and allied field $(\mathrm{p}<0.001)$ while doctors working in private were 0.107 times less likely to think that cadaveric dissection was unethical as compared to doctors working in public hospital $(\mathrm{p}<0.001)$.

\section{Discussion}

Anatomy in the past was considered as an important component of basic sciences in medical colleges and most of its teachings were based on detailed learning using cadaveric dissection. Being a very broad subject, it was soon realized that majority of its details were inapplicable and unnecessary in undergraduate programs because of poor long-term retention of information. This caused the conventional detailed curriculum to enfold into an applied knowledge form and the importance of anatomy as a major subject got reduced. Previously taught mainly using dissection augmented with didactic teaching and models [12], as the need of elaborate subject knowledge in clinical practice lessened, the use of dissection as its main mode of teaching was re-evaluated for benefits against high cost [13]. This led to the formation of two distinct schools of thoughts regarding the use of dissection as an anatomy teaching resource in medical colleges of Pakistan. The more financially equipped and government-sponsored teaching hospitals, due to ease of access to cadavers, continued using dissection as their main teaching tool augmented by prosection and didactic teaching; whereas, colleges funded privately discontinued it and replaced it with prosection, didactic teaching, models, computer-based programs and live surgeries. The purpose of this study was to assess the views of graduates from both college types regarding the better was of learning anatomy.

Students with access to dissection rated it to be less useful in comparison to computer-based programs and live surgeries. One reason could be the difficult student cadaver ratio of 50:1 that students have to counter in government-sponsored medical colleges along with the conditions of poorly ventilated, pungent smelling dissection halls [12]. According to our study, mean cadaveric dissection, prosection, model, computer model programs, and didactic teaching components were scored significantly higher by doctors working in private hospitals while live surgeries component was scored significantly higher by doctors in the public hospitals $(\mathrm{p}<0.001)$.

Previous studies concluded that learning anatomy was dependent more on memorization of organ systems and long lecture-based sessions with very little active student collaboration and applied experience involved, which led to most of the memorized knowledge acquired, lost by the time clinical experience began. However, in much accordance with previous study results [14], graduates largely believed that independent of the teaching resource used, instructors should make anatomy an interactive and involving subject with teamwork so the core concepts could be better understood and retained. A study also suggested that the end effect of a computer-based program was superior to the conventional ones used before and was also enjoyed by the students [15]. Incorporating lectures with role plays, animations, clinical cases in the form of problem-based learning result in active participation of students and hence may provide greater satisfaction and longer retention of core concepts in students. Although most methods (dissection and computer-based learning, live surgeries) have prevailed in medical colleges of Pakistan, neither were able to content students because the way these methods were used was similar - passive one-sided flow of information that usually resulted in a lack of interest in the subject and loss of concentration at the students end. Therefore, the preferred method chosen by doctors belonging to the two different college types was expecting it to be 
more interactive and fruitful, the one not experienced by them during their basic sciences years.

As the use of computer-based programs and related technologies as a teaching tool for anatomy rise around the world [16], its prospects of becoming a replacement for dissections in Pakistan was also addressed as part of this study and the response was analyzed in relation to gender. The result of this study also emphasized the global trend regarding the preferential use of technology, IT and software by male and female students in comparison to the conventional teaching methods. Our study showed that boys preferred the use of computer-based programs for educational purpose whereas girls favored conventional dissection as their choice of study tool. The reason for this difference was the greater accessibility of computers and software among boys as compared to girls in the country. Therefore, the ease of its use and operating confidence is higher in boys as compared to girls. Like most developing countries, access to computers and the internet is not easily available to students all around Pakistan. Therefore, similar to foreign study results, undergraduates in Pakistan also possess very limited knowledge of computers [17]. A study showed that the literacy level related to the use of the internet for accessing eHealth based information varied among female students [18]. Similar differences also prevail in Pakistan.

In our study, mean cadaveric dissection, prosection, and didactic teaching components were scored significantly higher by doctors in surgery and allied fields while live surgeries component was scored significantly higher by doctors in medicine and allied fields $(\mathrm{p}<0.001)$. A study conducted on Venezuelan surgeons concluded that $88 \%$ surgeon thought that cadaveric dissection was the most effective way to teach anatomy [19]. According to another study, 65\% surgeons selected "cadaver/prosection demonstration" as the most effective way to teach anatomy [20]. One study suggested that full-body dissection should be reserved for medical students who intend to pursue a surgical career while prosections and plastination are more suitable for medical students who intend to pursue a career in the medical and allied fields [13].

According to various studies, younger physicians were less likely to prefer cadaveric dissection as compared to their seniors [19]. In our study, a significant positive correlation was seen between age and cadaveric dissection method $\mathrm{p}<0.0005(\mathrm{r}=0.152)$. Among other tools of learning anatomy, the ethical aspect of deforming a human body and hence violating its sanctity has been brought into question time and time again. The view of many clinical physicians has not changed regarding the ethical aspect of cadaveric dissection. The majority of the surgical and allied specialties thought that it is ethical practice to perform dissection on cadavers. They put cadaveric dissection as the main method of learning and supported its use in their own clinical decision making.

However, to consider cadaveric dissection as a tool for learning ethics still remains a matter of personal opinion and its perception varies from personal beliefs to the religious upbringing of an individual [21-22]. To add another dimension, the method of acquiring the bodies is also another process not properly regulated in many locations and leaves room for more questions as to whether cadaveric dissection should give way to more recent and innovative methods of learning.

\section{Conclusions}

Dissection is still considered by several doctors as a valuable source of learning anatomy. However, the future of teaching anatomy does not depend on any single method. It is, in fact, the right combination of all available resources and using them in an interactive way that maximizes outcomes. 


\section{Additional Information \\ Disclosures}

Human subjects: Consent was obtained by all participants in this study. Shifa International Hospital, Institutional Review and Ethics Committee issued approval 433-282-2015. Animal subjects: All authors have confirmed that this study did not involve animal subjects or tissue. Conflicts of interest: In compliance with the ICMJE uniform disclosure form, all authors declare the following: Payment/services info: All authors have declared that no financial support was received from any organization for the submitted work. Financial relationships: All authors have declared that they have no financial relationships at present or within the previous three years with any organizations that might have an interest in the submitted work. Other relationships: All authors have declared that there are no other relationships or activities that could appear to have influenced the submitted work.

\section{References}

1. Williams AD, Greenwald EE, Soricelli RL, DePace DM: Medical students' reactions to anatomic dissection and the phenomenon of cadaver naming. Anat Sci Educ. 2014, 7:169-80.

10.1002/ase.1391

2. Azer SA, Eizenberg N: Do we need dissection in an integrated problem-based learning medical course? Perceptions of first- and second-year students. Surg Radiol Anat. 2007, 29:173-80. 10.1007/s00276-007-0180-X

3. Mwachaka PM, Mandela P, Saidi H: Repeated exposure to dissection does not influence students' attitudes towards human body donation for anatomy teaching. Anat Res Int. 2016, 2016:9251049. 10.1155/2016/9251049

4. Lempp HK: Perceptions of dissection by students in one medical school: beyond learning about anatomy. A qualitative study. Med Educ. 2005, 39:318-25. 10.1111/j.13652929.2005.02095.x

5. Winkelmann A: Anatomical dissection as a teaching method in medical school: a review of the evidence. Med Educ. 2007, 41:15-22. 10.1111/j.1365-2929.2006.02625.x

6. Cahill KC, Ettarh RR: Attitudes to anatomy dissection in an Irish medical school . Clin Anat. 2009, 22:386-91. 10.1002/ca.20777

7. Cho MJ, Hwang Y: Students' perception of anatomy education at a Korean medical college with respect to time and contents. Anat Cell Biol. 2013, 46:157-62. 10.5115/acb.2013.46.2.157

8. Memon IK: Anatomy education faces challenges in Pakistan . Anat Sci Educ. 2009, 2:193-4. 10.1002/ase.77

9. Sayyah M, Shirbandi K, Saki-Malehi A, Rahim F: Use of a problem-based learning teaching model for undergraduate medical and nursing education: a systematic review and metaanalysis. Adv Med Educ Pract. 2017, 8:691-700. 10.2147/AMEP.S143694

10. Yammine K, Violato C: A meta-analysis of the educational effectiveness of three-dimensional visualization technologies in teaching anatomy. Anat Sci Educ. 2015, 8:525-38.

10.1002/ase. 1510

11. Papa V, Vaccarezza M: Teaching anatomy in the XXI century: new aspects and pitfalls . Sci World J. 2013, 2013:310348. 10.1155/2013/310348

12. Qamar K, Osama M: Role of dissection in light of students' perceptions . J Pak Med Assoc. 2014, 64:1021-4.

13. Estai M, Bunt S: Best teaching practices in anatomy education: a critical review . Ann Anat. 2016, 208:151-157. 10.1016/j.aanat.2016.02.010

14. Burgess AW, Ramsey-Stewart G, May J, Mellis C: Team-based learning methods in teaching topographical anatomy by dissection. ANZ J Surg. 2012, 82:457-60. 10.1111/j.14452197.2012.06077.x

15. Nickel F, Hendrie JD, Bruckner T, et al.: Successful learning of surgical liver anatomy in a computer-based teaching module. Int J Comput Assist Radiol Surg. 2016, 11:2295-2301. 10.1007/s11548-016-1354-y

16. Kerby J, Shukur ZN, Shalhoub J: The relationships between learning outcomes and methods of teaching anatomy as perceived by medical students. Clin Anat. 2011, 24:489-97. 


\section{Cureus}

\subsection{2/ca.21059}

17. Ranasinghe P, Wickramasinghe SA, Pieris WR, Karunathilake I, Constantine GR: Computer literacy among first year medical students in a developing country: a cross sectional study. BMC Res Notes. 2012, 5:504. 10.1186/1756-0500-5-504

18. Stellefson M, Hanik B, Chaney JD, Tennant B: Analysis of ehealth search perspectives among female college students in the health professions using Q methodology. J Med Internet Res. 2012, 14:e60. 10.2196/jmir.1969

19. Romero-Reverón R: Venezuelan surgeons view concerning teaching human anatomical dissection. Anat Cell Biol. 2017, 50:12-16. 10.5115/acb.2017.50.1.12

20. Sheikh AH, Barry DS, Gutierrez H, Cryan JF, O'Keeffe GW: Cadaveric anatomy in the future of medical education: what is the surgeons view?. Anat Sci Educ. 2016, 9:203-8.

10.1002/ase. 1560

21. Snelling J, Sahai A, Ellis H: Attitudes of medical and dental students to dissection . Clin Anat. 2003, 16:165-72. 10.1002/ca.10113

22. Kerby J, Shukur ZN, Shalhoub J: The relationships between learning outcomes and methods of teaching anatomy as perceived by medical students. Clin Anat. 2011, 24:489-97.

10.1002/ca.21059 